\title{
Multi-figure Anatomical Objects for Shape Statistics
}

\author{
Qiong Han, Stephen M. Pizer, Derek Merck, Sarang Joshi, Ja-Yeon Jeong \\ Medical Image Display and Analysis Group \\ University of North Carolina at Chapel Hill, NC 27599, USA \\ han@cs.unc.edu
}

\begin{abstract}
Multi-figure m-reps allow us to represent and analyze a complex anatomical object by its parts, by relations among its parts, and by the object itself as a whole entity. This representation also enables us to gather either global or hierarchical statistics from a population of such objects. We propose a framework to train the statistics of multi-figure anatomical objects from real patient data. This training requires fitting multi-figure m-reps to binary characteristic images of training objects. To evaluate the fitting approach, we propose a Monte Carlo method sampling the trained statistics. It shows that our methods generate geometrically proper models that are close to the set of Monte Carlo generated target models and thus can be expected to yield similar statistics to that used for the Monte Carlo generation.
\end{abstract}

\section{Introduction}

The shape statistics of simple objects with one part have been widely studied. Methods using various representations have been proposed and shown to be effective $[1,2]$. However, many anatomical objects have multiple named parts, e.g., the prostate (fig. 1-a) has two seminal vesicles attached to it and the liver (fig. 1-b) has left and right lobes. Due to the inherent complexity of objects made from multiple parts, previous statistical descriptions of such objects concentrated on their global structure $[1,3]$ or on the extremely local behavior of geometric primitives, such as points, without reference to the parts' inter-relations $[4,5]$.

M-reps [6] have been successfully used to represent anatomical objects and complexes of objects [7-9]. An m-rep consists of one or more medial sheets, with the part corresponding to each sheet called a figure. Previous work on m-reps has been restricted to single figure objects. Computing statistics of such m-reps via principal geodesic analysis (PGA) $[2,14]$ has proved useful.

Medial description is also well suited to represent an object with parts [7, 10], e.g., an object with a protrusion subfigure, i.e., additive figure to the host (fig. 1-c), or an indentation subfigure, i.e., subtractive figure from the host (fig. 1-d). We use multi-figure m-reps to represent objects with multiple parts.

In the m-rep of a multi-figure object, each object part is geometrically represented by a single figure m-rep, and the figures of the object are connected by the hinge geometry briefly reviewed in section 2.2 . As with the single figure case, 


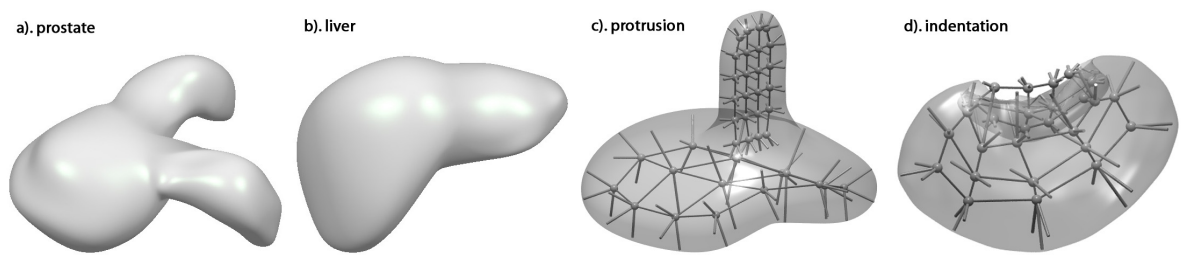

Fig. 1. a) A prostate with two seminal vesicle protrusions. b) A liver represented by the union of the left and right lobes. c) An object with protrusion. d) A kidney with the renal pelvis as an indentation subfigure. Object $a$ has three single-figure parts while objects $b$ - $d$ have two such parts.

the multi-figure m-rep describes an object at successively smaller scales following a coarse-to-fine hierarchy, for which the two top levels are 1) the object and 2) each individual figure and relations among the figures. In the top level the object is simply the union of its parts, enabling efficient analysis of the complex object as a whole. In addition, we can talk about individual part properties, such as shapes and volumes. Statistically, variation of the object within a population can be also measured in a multi-scale fashion. For example, we can investigate the variation of livers as well as of left liver lobes only.

In the process of training object statistics, we assume that each training object is given by a single binary characteristic image. We need to extract the m-rep for each object and then do PGA on the set of resulting m-reps. An efficient and reliable m-rep extraction method based on deformable model fitting is described in section 3 .

The multi-figure m-rep captures the natural hierarchy within a complex object. This form of representation also allows statistical analysis following the same hierarchy. While this approach can begin with global statistics on the union of the object parts, we describe a statistical description of the parts and their inter-relations via a hierarchical approach based on the residue. In section 4 we first sketch the global approach and then the residue approach. Both approaches are applied to the extracted multi-figure m-reps and the results are shown in section 5.1.

To evaluate the method of fitting m-reps to binary images, we propose a Monte Carlo technique and a means of data analysis based on geodesic differences between sample m-reps and the m-reps extracted from corresponding binary images. This data analysis method and its results are described in section 5.3.

We discuss incorporating the statistics into the training process to improve the quality of the extracted m-reps and conclude the paper in section 6 .

\section{$2 \quad$ Representing multi-figure objects}

In the multi-figure representation, each part of the object is represented by a single figure m-rep, which is briefly reviewed in the next subsection. 

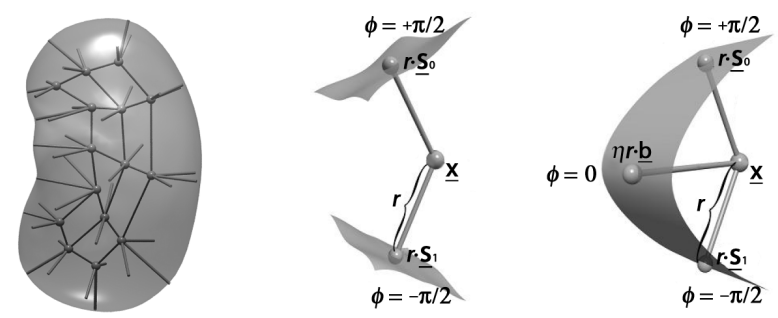

Fig. 2. Left: a single figure m-rep for a kidney and the object boundary implied by it. Middle: an internal medial atom. Right: an end atom. The local implied boundary is incident to and orthogonal to the spoke ends.

\subsection{Single figure $\mathrm{m}$-rep}

An m-rep is an extension of the Blum medial locus [11]; in the extension the medial locus forms the primitive description. The simplest geometric object is represented by a single continuous medial sheet with boundary. A discrete m-rep is formed by sampling the medial sheet over a spatially regular lattice to form a mesh of medial atoms (fig. 2-left), where each atom consists of a position on the medial sheet, and two equal length spokes. An internal medial atom is defined as a 4 -tuple $\left\{\underline{x}, r, \underline{s}_{0}, \underline{s}_{1}\right\}$, consisting of the hub position $\underline{x} \in \mathbb{R}^{3}$, the spoke length $r \in \mathbb{R}^{+}$, and the two spoke directions as two unit vectors $\underline{s}_{0}, \underline{s}_{1} \in S^{2}$ (fig. 2middle). The medial atoms on the edge of the medial sheet correspond to crests of the object boundary. Such an end atom adds a bisector spoke of length $\eta r$ with a corresponding crest sharpness parameter $\eta \in \mathbb{R}^{+}$(fig. 2-right). In section 3.1 we briefly review the mathematical background behind our representation.

Given an m-rep figure, a smooth object surface is generated to interpolate the boundary positions and normals implied by the atom spokes; presently a subdivision method [12] is used to generate the object boundary. If $u, v$ parametrizes the medial sheet, the implied boundary is parametrized by $(u, v, \phi)$, where $\phi$ designates the side of the figure from the top $\left(\phi=+\frac{\pi}{2}\right)$ to the bottom $\left(\phi=-\frac{\pi}{2}\right)$ and changes continuously across crests $\left(\phi \in\left[-\frac{\pi}{2},+\frac{\pi}{2}\right]\right)$ (fig. 2-right).

The single figure m-rep scheme has been extended to handle the complex of non-overlapping, single figure objects. Next we briefly review extending the representation to multi-figure m-rep objects.

\subsection{The multi-figure m-rep object with hinge geometry}

As detailed in [10], a multi-figure object is represented by a directed acyclic graph (DAG) of figures, each represented by a single figure m-rep. Subfigures can be recursively attached to their hosts to form any desired object DAG. This allows representation of arbitrarily complex objects, although most anatomical objects are adequately represented by a tree of two or three levels. The host and subfigure are determined according to anatomic naming and the tightness of posterior probabilities of the figures. In this paper we restrict our examples to objects with a single host figure and a single subfigure, e.g., the liver with the right lobe as the host and the left lobe as the subfigure. In the rest of this subsection we review how two figures are connected by the hinge geometry. Via 
the hinge the deformation of a host figure is propagated to its subfigure. A subfigure also has its own deformation which does not affect its host. A smooth surface boundary is then generated for the entire object by a method called blending.
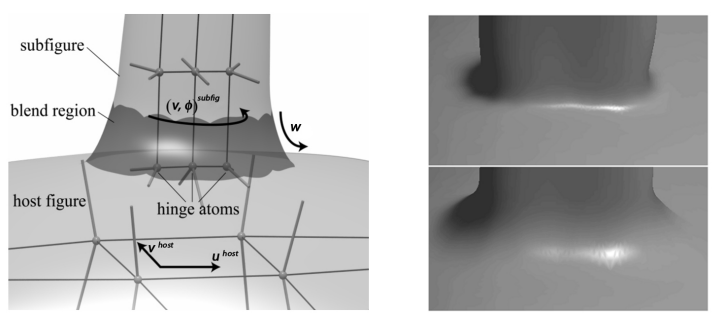

Fig. 3. Left: the host figure/subfigure arrangement, with the subfigure (six medial atoms appearing) on top, the host figure (four medial atoms showing) below, and the blend region shown darker. Right: different shapes of the blend region.

Hinge geometry The subfigure is attached to its host by a $1 D$ curve of hinge atoms, which, when sampled, form an end row or column of the subfigure atom mesh. Each hinge atom rides on the medially implied boundary of the host, with known figural coordinates of the host figure. The hinge geometry is an extension of the Blum medial locus that avoids the instability against boundary noise of the low-volume portion of branches. The host/subfigure arrangement is demonstrated in (fig. 3-left). The single hinge geometry allows both additive and subtractive subfigures (fig. 1-a-d).

With the two types of subfigure transformations below, we are able to represent and describe multi-figure objects with variable inter-figure relations.

Host figure implied subfigure transformation As the host figure deforms, the hinge atoms at the fixed $(u, v, \phi)$ in the host figure's coordinates change their locations and orientations. Since each subfigure atom can be represented as transformations of its neighboring atoms, the deformation of the host figure is propagated to the subfigure starting from the hinge atoms.

Hinge-relative subfigure transformations The subfigure can also translate, rotate, hinge, scale, and elongate on the host figure boundary while the host stays put. These basic hinge-relative transformations all take place in the host's figural coordinates and are at the subfigure scale levels. They form a key component of the coarse-to-fine hierarchy.

\subsection{Blending}

Blending, a well-studied field within computer graphics, is necessary if a smooth surface is to be generated from a host figure and its intersecting subfigure. To blend a subfigure with its host, an interpolating subdivision method is used to generate the implied boundary of each single figure. Each host figure and its 
attached subfigure meet and merge into each other. Designated sections from both figures are removed and replaced by a smooth region called the blend (fig. 3 -left). The blend between the two figures is parameterized by $(w, v, \phi)$, where $v$ and $\phi$ are the same as those in the subfigure coordinates and $w$ ranges from +1 , at the subfigure, to -1 , at the host. Two parameters delimiting the top and the bottom of the blend control the shape of the blend region (fig. 3-right).

\section{Fitting multi-figure m-reps to binary images}

The extraction of an m-rep from a binary characteristic image for statistical training is done by fitting a deformable m-rep template $M_{0}$ into the binary image. A large-scale-to-small optimization process over transformations associated with each respective stage is applied to the m-rep template. We define the objective function and then detail the transformations associated with each fitting stage in the following subsections. Firstly we review some mathematical background of the m-rep geometry; more details can be found in [2].

\subsection{Background theory review}

As the primitive in an m-rep, each internal(end) medial atom can be understood as a point on the manifold $\mathcal{M}_{\text {int }}(1)\left(\mathcal{M}_{\text {end }}(1)\right)=\mathbb{R}^{3} \times \mathbb{R}^{+} \times S^{2} \times S^{2}\left(\times \mathbb{R}^{+}\right)$. Let $\mathcal{M}(1)$ denote the manifold for a medial atom without specifying whether it is an internal or end atom. Thus an m-rep of $n$ medial atoms can be seen as a point on the manifold $\mathcal{M}(n)=[\mathcal{M}(1)]^{n}$.

The space $\mathcal{M}(n)$ is a particular type of manifold known as a Riemannian symmetric space, which simplifies the calculation of geodesics and distances. Let $\operatorname{dis}(y, z): \mathcal{M}(n) \times \mathcal{M}(n) \rightarrow \mathbb{R}^{+} \cup\{0\}$ denote the geodesic distance, i.e., the locally shortest distance on the manifold $\mathcal{M}(n)$, between two points $y, z \in \mathcal{M}(n)$. There are a pair of maps $\operatorname{Exp}_{y}$ and $\log _{y}$ that map between $\mathcal{M}(n)$ and the tangent space $T_{y} \mathcal{M}(n)$ at $y$, and are inverse of each other. $T_{y} \mathcal{M}(n)$ can be identified with $\mathbb{R}^{8 n+n_{\text {ext }}}$ with $n_{\text {ext }}$ as the number of end atoms in $y$ and $z$.

- $\log _{y}(z)$ maps the point $z$ to the tangent space $T_{y} \mathcal{M}(n)$ at $y$. The geodesic distance between $y$ and $z$ is preserved and calculated via the Log map.

$$
\operatorname{dis}(y, z)=\left\|\log _{y}(z)\right\|
$$

- $\operatorname{Exp}_{y}(\underline{v})$ maps the tangent vector $\underline{v} \in T_{y} \mathcal{M}(n)$ to the point on $\mathcal{M}(n)$ along the geodesic curve $\gamma_{\underline{v}}(t)$. The distance is preserved as $\operatorname{dis}\left(y, \operatorname{Exp}_{y}(\underline{v})\right)=\|\underline{v}\|$.

Given dis, we can calculate the Fréchet mean $\bar{M}$ of $N$ points (m-reps) $\left\{M_{i} \mid M_{i} \in\right.$ $\mathcal{M}(n), i=1,2, \ldots, N\}$ by minimizing the average squared geodesic distance:

$$
\bar{M}=\operatorname{Mean}\left(M_{i}\right)=\underset{M \in \mathcal{M}(n)}{\arg \min } \frac{1}{N} \sum_{i=1}^{N}\left\|\log _{M}\left(M_{i}\right)\right\|^{2}
$$

In the residue approach described in section 4.2 , we need to calculate the difference between m-reps via the difference between their corresponding atoms. Let $\underline{a}_{1}, \underline{a}_{2} \in \mathcal{M}(1)$ be two corresponding atoms. Then their difference is 


$$
\underline{a}_{1} \ominus \underline{a}_{2}=g_{\underline{a}_{2}}^{-1} \circ \underline{a}_{1} \in \mathcal{M}(1),
$$

where $g_{\underline{a}_{2}}^{-1} \in G(1)$ is the composition of hub translation, spoke magnification(s), and spoke rotations determining an atom transformation and, $G(1)$ denotes the Lie-group of such transformations.

Assume an m-rep template $\in \mathcal{M}(n)$ has $n$ medial atoms $\left\{\underline{a}_{i}\right\} . G(n)=[G(1)]^{n}$ acts smoothly on $\mathcal{M}(n)$ as the transformation between m-reps. The difference between two m-reps $M_{1}, M_{2} \in \mathcal{M}(n)$ from the same template is defined as

$$
M_{1} \ominus M_{2}=\prod_{j=1}^{n}\left(\underline{a}_{1 j} \ominus \underline{a}_{2 j}\right) \in \mathcal{M}(n)
$$

\subsection{Objective function}

The objective function measuring the mismatch between the m-rep and binary image [15] is a sum of three terms: an m-rep-to-binary boundary distance, a term penalizing irregularity of the m-rep atoms, and a term for achieving correspondence across the m-reps in a training population.

Binary image match A distance map image $D(\underline{x}): \mathbb{R}^{3} \rightarrow \mathbb{R}^{+} \cup\{0\}$ is calculated for each given binary image $I_{b}$ by an extension of the Danielsson distance mapping [13] to 3D. The binary image match term is then calculated by the integral of the distance map on the m-rep implied object surface $\mathcal{B}$, except that at the boundary locations where the surface normal differs from the distance gradient by more than a certain threshold, $D(\underline{x})$ is replaced by the distance along the surface normal to the nearest binary object boundary location. $\mathrm{L}(M, D)$ measures how well $M$ fits into the distance map image $D$.

$$
\mathrm{L}(M, D)=\alpha \cdot \frac{1}{\operatorname{area}(\mathcal{B}(M))} \int_{\mathcal{B}(M)} D^{2}(\underline{x}) d^{2} A
$$

Regularity penalty This term penalizes non-uniform spacing and changes in spoke length and orientation of the medial atom. It leads to proper object geometry and correspondence across the training cases.

$$
\operatorname{Reg}(M)=\beta \cdot \sum_{i=1}^{n} \| \log _{\underline{a}_{i}}\left(\operatorname{Mean}\left(\mathrm{N}\left(\underline{a}_{i}\right)\right) \|^{2}\right.
$$

For each medial atom $\underline{a}_{i}$, the regularity is calculated as the squared geodesic distance between $\underline{a}_{i}$ and the Fréchet mean (eqn. 2) of its neighboring atoms $\mathrm{N}\left(\underline{a}_{i}\right)$. The penalties are then accumulated for all the medial atoms of the object.

Correspondence to a reference $\mathbf{m}$-rep The reference penalty depends on the geodesic distance between the current $M$ and the reference m-rep $M_{0}$, which the fitting starts with in our present implementation. This term explicitly penalizes weak correspondence across m-reps.

$$
\operatorname{Ref}(M)=(1-\alpha-\beta) \cdot\left\|\log _{M_{0}}(M)\right\|^{2}
$$


In equations (5)-(7), $\alpha, \beta \geqslant 0$, and $\alpha+\beta \in[0,1]$. The complete objective function is the combination of the three terms:

$$
\operatorname{Obj}(M, D)=\mathrm{L}(M, D)+\operatorname{Reg}(M)+\operatorname{Ref}(M)
$$

A two-figure m-rep is used as the example in the following subsections. Assume a two-figure m-rep template $M_{0}$ has host figure $F_{1}$ and subfigure $F_{2}$, and each figure $F_{i} \subset M_{0}$ has atoms $\left\{\underline{a}_{j}^{i} \mid i=1,2, j=1,2, \ldots, n_{i}\right\}$.

\subsection{Extraction framework}

The objective function is then optimized over the following sequence of transformations, successively finer in scale, applied to the m-rep template.

- Initial alignment of $M_{0}$ by $T_{1} \in \mathbb{R}^{3} \times \mathbb{R}^{+} \times S O(3)$, calculated by the template $M_{0}$ and the distance map image $D$;

- object stage: $T_{o b j} \in \mathbb{R}^{3} \times \mathbb{R}^{+} \times S O(3)$, on the entire object;

- host figure: the host $F_{1}$ is the target and the subfigure is deformed by an implied transformation $T_{\text {host_implied }} \in \mathbb{R}^{3} \times \mathbb{R}^{+} \times S O(3)$;

- figural stage: $T_{\text {host_fig }} \in \mathbb{R}^{3} \times \mathbb{R}^{+} \times S O(3)$, on the host figure;

- atom stage: $T_{\text {host_atom }} \in G\left(n_{1}\right)$, on the host figure atoms $\underline{a}_{1,2, \ldots, n_{1}}^{1}$;

- subfigure stage: the subfigure $F_{2}$ is the target in this stage;

- figural stage: $T_{\text {sub_fig }} \in \mathbb{R}^{3} \times \mathbb{R}^{+} \times S O(3)$, on the subfigure. At the end of this stage, the hinge atoms are projected onto the host figure surface;

- atom stage: $T_{\text {sub_atom }} \in G\left(n_{2}\right)$, on the subfigure atoms $\underline{a}_{1,2, \ldots, n_{2}}^{2}$.

input:

a two-figure m-rep template $M_{0}$ with host figure $F_{1}$ and subfigure $F_{2}$;

a distance map images $D_{i}$ : calculated from the given binary images. $I_{b i}$.

output:

extracted two-figure m-reps $M_{i}$ from the images $D_{i}$.

\section{framework:}
for each $D i\{$
1. Calculate $T_{1}$ by the 1 st and 2 nd moments of $M_{0}$ and $D_{i}, M_{1}=T_{1} \circ M_{0}$;
2. $T_{2}=\arg \min _{T_{o b j}}\left(\operatorname{Obj}\left(T_{o b j} \circ M_{1}, D_{i}\right)\right), M_{2}=T_{2} \circ M_{1}$;
3. $T_{3}=\arg \min _{T_{\text {host fig }}}\left(\operatorname{Obj}\left(T_{\text {host_fig }} \circ F_{1} \subset M_{2}, D_{i}\right)\right), M_{3}=T_{3} \circ M_{2}$;
4. $T_{4}=\arg \min _{T_{\text {host_atom }}}\left(\operatorname{Obj}\left(T_{\text {host_atom }} \circ F_{1} \subset M_{3}, D_{i}\right)\right), M_{4}=T_{4} \circ M_{3}$;
5. $T_{4}^{\prime}=T_{\text {host_implied }}, M_{4}^{\prime}=T_{4}^{\prime} \circ F_{2} \in M_{4}$;
6. $T_{5}=\arg \min _{T_{\text {sub } b_{-} \text {ig }}}\left(\operatorname{Obj}\left(T_{\text {sub } f i g} \circ F_{2} \subset M_{4}^{\prime}, D_{i}\right)\right), M_{5}=T_{5} \circ M_{4}^{\prime}$
7. $M_{5}^{\prime}=$ hinge atoms in $F_{2} \subset M_{5}$ are projected to the surface of $F_{1} \subset M_{5}$;
8. $T_{6}=\arg \min _{T_{\text {sub_atom }}}\left(\operatorname{Obj}\left(T_{\text {sub_atom }} \circ F 2 \subset M_{5}^{\prime}, D_{i}\right)\right), M_{i}=T_{6} \circ M_{5}^{\prime}$;
\}

This framework can be extended to arbitrary levels of hierarchy. However in this paper, our data and experiments focus on objects with two-figures. Next we describe the statistical analysis on the extracted multi-figure m-reps. 


\section{Statistics of multi-figure objects}

As reviewed in section 3.1, an m-rep consisting of $n$ atoms is a point on the manifold $\mathcal{M}(n)$. The principal geodesic analysis has been proposed to do statistical analysis for single figure object in such a space [2]. Briefly, given $N$ m-reps $\left\{M_{i} \mid M_{i} \in \mathcal{M}(n)\right\}$, the Fréchet mean $\bar{M}$ is first calculated using (2). Let $u_{i}=$ $\log _{\bar{M}}\left(M_{i}\right)$, then the covariance matrix is given by $\Sigma=\frac{1}{N} \sum_{i=1}^{N} u_{i} u_{i}^{T}$. The PGA is computed as $\left\{\underline{p}_{k}, \lambda_{k} \mid \underline{p}_{k} \in T_{\bar{M}} \mathcal{M}(n)\right.$ are the principal geodesic directions, $\lambda_{k} \in$ $\mathbb{R}$ are the variances $\}=\{$ eigenvectors/eigenvalues of $\Sigma\}$.

\subsection{Global statistics}

Assume that a multi-figure object $O$ has $N$ figures as $\left\{F_{i}, i=1,2, \ldots, N\right\}$ and each figure $F_{i}$ has $n_{i}$ medial atoms. Treat $O$ as the union of all its figures and let $n_{O}$ be the total number of atoms in $O$. The global statistics of such objects are computed by the mean object $\bar{O}$ and the PGA in $\mathcal{M}\left(n_{O}\right)$.

\subsection{Hierarchical statistics based on residue}

For multi-figure m-rep statistics we follow the hierarchical statistical framework for multi-objects detailed in [14]. In the case of two-figure object $O$ consisting of figures $F_{1}$ and $F_{2}$ with $n_{1}, n_{2}$ atoms, respectively, the host and subfigure are like the single figure objects in the complexes of multi-objects, and the hinge atoms act as the augmenting atoms that relate the host figure's changes to the sympathetic subfigure changes. Let $n_{O}=n_{1}+n_{2}$. Three definitions are needed to sketch how two-figure object statistics are represented and computed.

- Residue: difference between two m-reps by the operation $\ominus$ (eqn. 3,4);

- Augmentation: $U_{1}=F_{1} \cup A_{1}$ denotes the union of host figure atoms and the hinge atoms $A_{1}$ in the subfigure $F_{2}$;

- Projection: an m-rep $M$ can be projected into the PGA subspace by $\pi_{H}(M) \approx$ $\operatorname{Exp}_{\bar{M}} \sum_{i=1}^{k}\left\langle\underline{p}_{i}, \log _{\bar{M}}(M) \underline{p}_{i}\right.$.

There are three parts $\mathrm{PGA}_{g}, \mathrm{PGA}_{h}$, and $\mathrm{PGA}_{s}$ in the hierarchical statistics for a two-figure object.

1. $\mathrm{PGA}_{g}$ : statistics on the $n_{O}$ atoms making up the entire object. This captures the global shape variation of the object. This variation is removed from both the host figure atoms and the subfigure atoms before steps 2 and 3 ;

2. $\mathrm{PGA}_{h}$ : statistics on the residue of the union $U_{1}$ of the host figure atoms and the hinge atoms in the subfigure. This describes the remaining variation of $U_{1}$ after the projection to the global variation $\mathrm{PGA}_{g}$ has been removed;

3. $\mathrm{PGA}_{s}$ : statistics on the residue of subfigure $F_{2}$ after the residual changes in the host figure are propagated to the subfigure and have then been removed. The variation in the host figure is computed by projection to $\mathrm{PGA}_{h}$, and the propagation is computed via the hinge atoms.

We applied both the global and the hierarchical statistical analysis to the extracted m-reps of livers. The results are shown in the next section. 


\section{Results and evaluation}

\subsection{The data and results}

We use 15 expert segmented binary images of livers. A two-figure m-rep template is fit into the images by the framework described in section 3 . There are $3 \times 7$ and $3 \times 4$ sampled atoms in the host figure and subfigure, respectively. Three atoms in the subfigure are used as the hinge atoms. The total of 33 liver atoms lie in a manifold of 290-dimensions. The extracted m-reps $M_{1,2, \ldots, 15}$ are used for the shape statistics. Fig. 4-left shows the cumulative variances in the principal modes of the global statistics; 4-right shows the variations of the livers in the host and subfigure residue statistics as parts of the hierarchical statistics.
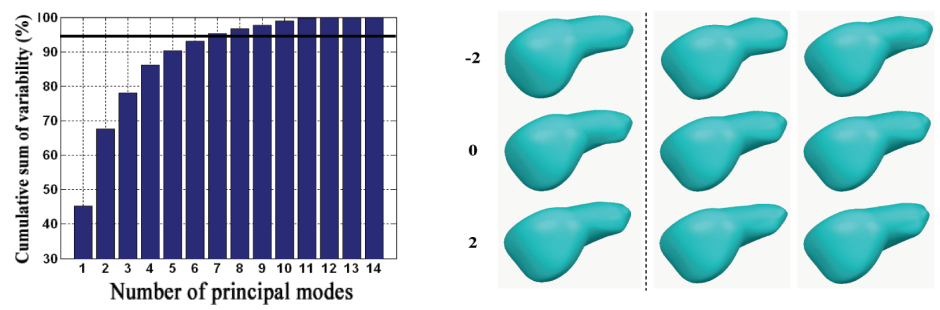

Fig. 4. Left: accumulated sum of the variances from the global stats $\mathrm{PGA}_{g}$ : the first $\mathbf{7}$ modes capture over $\mathbf{9 5 \%}$ of the total variability. Right: the residue shape variation after the global variation is removed: each column shows the liver -2 standard deviations from the residue mean along the respective eigenmode, the residue mean, and the liver +2 standard deviations from the mean. The left column shows the first principal mode of the host residue stats $\mathrm{PGA}_{h}$; the other two columns show the first two modes of the subfigure residue stats: $\mathrm{PGA}_{s}$ describes the remaining shape variation of the subfigure after the global and host-implied variation have been removed.

\subsection{Generate new m-reps using the Monte Carlo method}

In order to evaluate the extraction method, we need binary images for which we know the true m-reps. A sampling scheme based on a Monte Carlo method, described next, is used to generate sample m-reps from the trained statistics. Sample binary images used as target images are then created as the interior of the sampled m-reps.

Assume the PGA statistics on the extracted training m-reps (with $n$ atoms) are the mean m-rep $\bar{M}$, the first $N_{P G A}$ principal variances $\left\{\lambda_{1,2, \ldots, N_{P G A}}\right\}$, and the corresponding first $N_{P G A}$ normalized principal geodesic directions $\left\{\underline{p}_{1,2, \ldots, N_{P G A}}\right\}$, which is a subset of all the principal directions and sufficient to describe the variability of the m-rep shape space. New m-reps are generated by using the PGA as the population distribution $p(M)$ and sampling from it via the Monte Carlo method.

1. Generate a Gaussian vector $\underline{\alpha}=\left(\alpha_{1,2, \ldots, N_{P G A}}\right)$, with each $\alpha_{i}$ sampled from the standard normal distribution $\mathcal{N}(0,1)$;

2. Apply $\underline{\alpha}$ as the components on the principal directions for a tangent vector $\underline{v}=\sum_{i=1}^{\bar{N}_{P G A}} \alpha_{i} \sqrt{\lambda_{i}} \cdot \underline{p}_{i}$ in the tangent space $T_{\bar{M}}(\mathcal{M}(n))$ at the mean $\bar{M}$; 
3. The exponential map is used to map $\underline{v}$ to the m-rep manifold as a sampled m-rep $M=\operatorname{Exp}_{\bar{M}}(\underline{v})$.

\subsection{Evaluation}

The diagram in fig. 5 details this evaluation using the Monte Carlo sample generation described in the previous section 5.2.

\begin{tabular}{|c|c|}
\hline$p(M)$ & $\begin{array}{l}\text { The Monte Carlo method is used to generate } N \text { target } \mathbf{m} \text { - } \\
\text { reps } M_{i} \text {; }\end{array}$ \\
\hline$M_{i}, i=1,2, \ldots, N$ & \multirow{3}{*}{$\begin{array}{l}\text { Danielsson distance mapping, from the implied boundary } \\
\text { of each sampled } M_{i} \text {, is used to generate the target binary } \\
\text { image } I_{i} \text { of the interior of } M_{i} \text {; }\end{array}$} \\
\hline (2)- & \\
\hline$I_{i}, i=1,2, \ldots, N$ & \\
\hline (3)- & \multirow{2}{*}{$\begin{array}{l}\text { Deform the mean m-rep } \bar{M} \text { into each target image } I_{i} \text { using } \\
\text { the extraction method. The extracted m-rep is } M_{i}^{\prime} \text {; }\end{array}$} \\
\hline$M_{i}^{\prime}, i=1,2, \ldots, N$ & \\
\hline$\frac{1}{\frac{1}{N} \sum_{i=1}^{N}\left\|\log _{M_{t}}\left(M_{t}^{\prime}\right)\right\|^{2}}$ & $\begin{array}{l}\text { The geodesic distance }\left\|\log _{M_{i}}\left(M_{i}^{\prime}\right)\right\| \text { is then used as the } \\
\text { measure of the extraction quality. }\end{array}$ \\
\hline
\end{tabular}

Fig. 5. Diagram flow to evaluate the extraction process given an initial population distribution $p(M)$

50 liver m-reps (fig. 6-left) were generated using the Monte Carlo sampling method. The evaluation results are shown in fig. 6-middle as a histogram of the mismatch (geodesic distance) between the extracted m-reps and their corresponding m-reps as the truth, which the target images are created from. The average geodesic distance across all the livers is 0.054 , in the units of the average boundary displacement implied by all the atoms together. And the averaged m-rep-to-binary distance is 0.674 image voxel for all the $50 \mathrm{~m}$-reps.
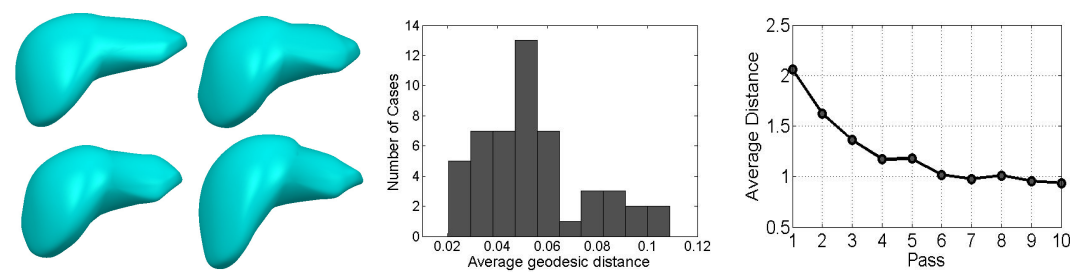

Fig. 6. Left: $\mathbf{4}$ of the $\mathbf{5 0}$ sampled m-reps used in the evaluation. Middle: evaluation results of the extraction framework shown as a histogram of geodesic distances between the extracted m-reps $M_{i}^{\prime}$ and the m-reps $M_{i}$ as the truth. Right: in the first ten passes of the multi-pass extraction using the shape statistics, the fitting quality improves while the average distance from the m-rep implied surface points to the closest contour points in the binary image decreases. The distance is in the unit of image voxel. 


\section{Discussion and conclusion}

Our examples suggest that extracted m-reps are good enough to be useful in applications requiring statistical analysis, such as segmentation by the posterior optimization of m-reps or the characterization of the geometric differences between object populations.

We have observed that by the incorporation of the PGA statistics into a multi-pass training, the fitting quality can be improved. The first pass uses the same method described in section 3.3 to extract the m-reps. A following new pass uses the shape statistics trained on the extracted m-reps from the previous pass as the shape prior. Assume the PGA from a previous pass is given by $\left\{\bar{M}, \lambda_{j}, \underline{p}_{j}\right\}$. In a new pass, m-reps are extracted from the same images by the optimization over the coefficients of the principal directions in the following objective function, combining the object-to-image mismatch and the squared Mahalanobis distance as the present log shape prior.

$$
\underset{\left(\alpha_{1}, \alpha_{2}, \ldots, \alpha_{N_{p g c}}\right)}{\arg \min } \mathrm{L}\left(M_{i}=\operatorname{Exp}_{\bar{M}}\left(\sum_{j=1}^{N_{P G A}} \alpha_{j} \sqrt{\lambda_{j}} \cdot \underline{p}_{j}\right), D_{i}\right)+\sum_{j=1}^{N_{P G A}} \frac{\alpha_{j}^{2}}{\lambda_{j}}
$$

Results (fig. 6-right) indicate that the first several passes of the fitting with statistics improve the quality of the extracted m-reps. $Y$ axis in fig. 6-right is the average image match distance (defined in section 3.2) over all the 15 images. The decreasing distance in the first 10 passes indicates the improvement of the extraction. However, the convergence of this process is still under research.

We have shown a framework to extract the medial descriptions represented by multi-figure m-reps from binary characteristic images of multi-figure objects, especially the objects with two-figures as demonstrated in the result section 5.1. A Monte Carlo method has been designed to evaluate the extraction process. We have also shown how to do either global or hierarchical statistical analysis on multi-figure objects. We are evaluating our method when applied to the objects represented by a tree of more than one subfigures (fig. 1-a), as well as the objects also with indentation subfigure(s) (fig. 1-d). The bias and reliability of the statistical framework and the convergence of the multi-pass fitting are also subjects of research.

\section{Acknowledgement}

We thank Keith Muller for advice on Monte Carlo generation of m-reps and analysis of their results, P. Thomas Fletcher, Conglin Lu, and Rohit Saboo for m-reps statistics and Monte Carlo methodology, Stephen Aylward and the CADDLab in Radiology Department of UNC for the liver binary characteristic images, Delphi Bull for the help on organizing the reference list. The work reported here was done under the partial support of NIH grant P01 EB02779. 


\section{References}

1. Cootes, T.F., Taylor, C.J., Cooper, D.H., and Graham, J.: Active shape models their training and application. Computer Vision and Image Understanding. 61(1): 38-59, 1995.

2. Fletcher, P.T., Lu, C., Pizer, S.M., and Joshi, S.: Principal geodesic analysis for the nonlinear study of shape. IEEE Transactions on Medical Imaging (TMI), 23(8): 995-1005, Aug 2004.

3. Gerig, G., Styner, M., Weinberger, D., Jones, D., and Lieberman, D. : Shape analysis of brain ventricles using SPHARM. IEEE Workshop on Mathematical Methods in Biomedical Image Analysis (MMBIA), 171-178, 2001.

4. Csernansky, J.C., Joshi, S., Wang, L., Gado, M., PhilipMiller, J., Grenander, U., and Miller, M.I.: Hippocampal morphometry in schizophrenia by high dimensional brain mapping. National Academy of Science, 95: 11406-11411, Sept 1998.

5. Styner, M., Gerig, G., Lieberman, J., Jones, D., and Weinberger, D.: Statistical shape analysis of neuroanatomical structures based on medial models. Medical Image Analysis (MEDIA), 7(3): 207-220, 2003.

6. Pizer, S.M., Fletcher, T., Fridman, Y., Fritsch, D.S., Gash, A.G., Glotzer, J.M., Joshi, S., Thall, A., Tracton, G., Yushkevich, P., and Chaney, E.L.: Deformable mreps for $3 \mathrm{D}$ medical image segmentation. Int. J. Comp. Vis. - Special UNC-MIDAG issue (IJCV), 55(2): 85-106, Nov-Dec 2003.

7. Pizer, S.M., Fletcher, P.T., Joshi, S., Gash, A.G., Stough, J., Thall, A., Tracton, G., and Chaney E.L.: A method and software for segmentation of anatomic object ensembles by deformable m-reps. To appear, Medical Physics, 2005.

8. Rao, M., Stough, J., Chi, Y-Y., Muller, K., Tracton, G.S., Pizer, S.M., and Chaney E.L.: Comparison of human and automatic segmentations of kidneys from CT images. Int. J. Rad. Onc., Biol., Physics, 61(3): 954-960, 2005.

9. Chaney, E.L., Pizer, S.M., Joshi, S., Broadhurst, R., Fletcher, R., Gash, G., Han, Q., Jeong, JY., Lu, C., Merck, D., Stough, J., Tracton, G., MD Bechtel, J., Rosenman, J., Chi, YY., and Muller, K.: Automatic male pelvis segmentation from CT images via statistically trained multi-object deformable m-rep models. Abstract and presentation at Annual Meeting of American Society for Therapeutic Radiology and Oncology (ASTRO), 2004.

10. Han, Q., Lu, C., Liu, S., Pizer, S.M., Joshi, S., and Thall, A.: Representing multifigure anatomical objects. IEEE International Symposium on Biomedical Imaging (ISBI), 1251-1254, 2004.

11. Blum, H. and Nagel, R.: Shape description using weighted symmetric axis features. Pattern Recognition, 10: 167-180, 1978.

12. Thall, A.: Fast $C^{2}$ interpolating subdivision surfaces using iterative inversion of stationary subdivision rules. http://midag.cs.unc.edu/pub/papers/Thall TR02001.pdf, 2002.

13. Danielsson, P.E.: Euclidean distance mapping. Computer Graphics and Image Processing, 14: 227-248, 1980.

14. Pizer, S.M., Jeong, J., Lu, C., Muller, K. and Joshi, S.: Estimating the statistics of multi-object anatomic geometry using inter-object relationships. Proc. Workshop on Deep Structure, Singularities and Computer Vision, Springer LNCS, 2005.

15. Merck, D., Gash, G., Joshi S., and Pizer, S.M: On single figure statistical m-rep model construction. Submitted for publication, http://midag.cs.unc.edu/GeomModFS.html, 2005. 